\title{
Valorização agrícola de lamas - disponibilidade do fósforo
}

\section{Agricultural valorization of sewage sludge - phosphorus availability}

\author{
Inês Bártolo*1, Rui Fernandes² e Cristina Sempiterno² \\ ${ }^{1}$ Faculdade de Ciências e Tecnologia, Universidade Nova de Lisboa. Departamento de Ciências e Engenharia do Ambiente. Campus de Caparica, 2829-516 Caparica, Portugal \\ 2INIAV - Instituto Nacional de Investigação Agrária e Veterinária. Unidade Estratégia de Investigação e Serviços de Sistemas Agrários e Florestais e Sanidade Vegetal. Tapada da Ajuda, \\ Apartado $32281301-903$ Lisboa, Portugal \\ (*E-mail: inesmbartolo@gmail.com) \\ http://dx.doi.org/10.19084/RCA17080
}

Recebido/received: 2017.03.30

Recebido em versão revista/received in revised version: 2017.05 .04

Aceite/accepted: 2017.05.04

\section{R E S U M O}

As lamas das Estações de Tratamento de Águas Residuais (ETARs) contêm matéria orgânica e nutrientes indispensáveis ao crescimento e desenvolvimento das plantas que interessará aproveitar na fertilização das culturas permitindo, assim, uma economia no uso de adubos minerais. Com o objetivo de contribuir para um aumento do conhecimento sobre o fornecimento de fósforo ao solo e às culturas nele instaladas através da aplicação de lamas de ETAR, realizou-se um ensaio em vasos, com milho, delineado em blocos completos casualizados, com três repetições e treze tratamentos experimentais: um tratamento testemunha, sem aplicação de fósforo; quatro tratamentos com níveis crescentes de fósforo veiculados pela lama; quatro tratamentos com níveis crescentes de fósforo veiculados por fosfato de cálcio; quatro tratamentos com os mesmos níveis crescentes de fósforo veiculados pela mistura de lama e fosfato de cálcio. A produção de biomassa foi mais elevada nos tratamentos que receberam as quantidades mais elevadas de fósforo veiculado pelo adubo mineral e pela mistura deste com lama. A aplicação da quantidade máxima de lama (equivalente a $120 \mathrm{t} \mathrm{ha}^{-1}$ ) provocou um decréscimo na produção de biomassa e conduziu a um menor aproveitamento de fósforo pelas plantas, o que reforça a necessidade de estabelecer limites máximos para as quantidades de lama a aplicar ao solo agrícola. Os resultados favoráveis, a nível da produção de biomassa e da recuperação aparente de fósforo, obtidos com a mistura de lama com o adubo mineral, permitiram concluir que a lama poderá possibilitar uma economia no uso daqueles fertilizantes, substituindo em parte a sua utilização.

Palavras-chave: fósforo, lamas de depuração, recuperação, remoção.

\section{A B S T R A C T}

Sewage sludge from treatment plant are rich in organic matter and nutrients, which are essential to the growth and development of plants. When properly applied to the soil, they contribute to fertilization of crops, thus saving the use of chemical fertilizers. In order to contribute to a better understanding of sludge phosphorus supply to the soil, a pot trial was installed using maize as a test plant. For this purpose, the experiment was arranged into randomized complete block design, with three replicates, and thirteen experimental treatments as follows: (i) a control treatment without phosphorus supply; (ii) four treatments with increasing levels of phosphorus supplied through sludge; (iii) four treatments with increasing levels of phosphorous supplied through calcium phosphate and (iv) four treatments with the same levels of phosphorus but supplied through sludge and calcium phosphate.

The biomass production was higher with the treatments that received the higher amounts of phosphorus supplied through sludge and calcium phosphate. The supply of the maximum amount of sludge (equivalent to $120 \mathrm{t} \mathrm{ha}^{-1}$ ) caused a decrease of the biomass production and led to a lower use of phosphorus by the plants. Therefore, it is necessary to establish maximum limits for the quantities of sludge to be supplied to the agricultural soils. In relation to the production of biomass and the apparent recovery of phosphorus, the good results show that the combination of sludge and calcium phosphate will be able to make possible an economy of these fertilizers. Even though sludge cannot totally replace phosphate fertilizers, it can be used as a partial substitute.

Keywords: phosphorus, sewage sludge, recovery, removal. 


\section{INTRODUÇÃO}

As lamas provenientes das ETAR são caracterizadas por serem ricas em matéria orgânica e conterem elevados teores de azoto e de fósforo, que lhes conferem valor agronómico (Gonçalves, 2005). A quantidade e qualidade das lamas dependem da eficácia do tratamento e da carga poluente do efluente tratado e, também, das características técnicas do processo de tratamento de águas residuais (Fytili e Zabaniotou, 2008).

As lamas de depuração, que satisfaçam todos os requisitos impostos na legislação (Diretiva n. 86/278/CEE, do conselho, de 12 de junho e DL n.. 276/2009, de 2 de outubro), são valiosos fertilizantes que, se aplicados corretamente aos solos, não trarão praticamente riscos para a saúde pública e para o ambiente (Soveral-Dias, 2004).

Atualmente deparamo-nos com o problema de a maior parte dos sistemas de produção agrícola se encontrarem dependentes da aplicação de fertilizantes fosfatados, derivados de fosforite, sendo este um recurso finito que poderá esgotar-se em anos vindouros. São, por isso, necessárias fontes alternativas de fósforo para garantir que os agricultores têm acesso, a longo prazo, a fertilizantes fosfatados em quantidades suficientes, para produzir alimentos, sem comprometer o ambiente (Cordell e White, 2014).

O presente trabalho, desenvolvido no Instituto Nacional de Investigação Agrária e Veterinária, I.P. (INIAV), teve como objetivo avaliar as quantidades de fósforo disponibilizadas à cultura do milho (Zea mays L.) através da aplicação de quantidades crescentes de lama da ETAR de Beirolas, a um solo agrícola. Comparam-se os resultados obtidos com os resultantes da aplicação de fósforo somente na forma mineral (fosfato de cálcio) e os resultantes da aplicação da mistura de lama com o citado adubo. Mediu-se a resposta em termos de produtividade e da quantidade de fósforo removida e recuperada pela planta teste.

\section{MATERIAIS E MÉTODOS}

A macro amostra de terra utilizada no ensaio em vasos era proveniente da camada superficial

Quadro 1 - Características físico-químicas da macroamostra de terra utilizada no ensaio

\begin{tabular}{|c|c|c|c|c|c|}
\hline Parâmetros & & & Parâmetros & & \\
\hline Areia & $(\%)$ & 92,3 & Acidez de troca & $\left(\mathrm{cmol}(+) \mathrm{kg}^{-1}\right)$ & 1,1 \\
\hline Limo & $(\%)$ & 4,9 & SBT & $\left(\mathrm{cmol}^{(+)} \mathrm{kg}^{-1}\right)$ & 0,58 \\
\hline Argila & $(\%)$ & 2,9 & CTC & $\left(\mathrm{cmol}^{(+)} \mathrm{kg}^{-1}\right)$ & 1,68 \\
\hline Classificação textural & & Arenosa & GSB & $(\%)$ & 34,7 \\
\hline $\mathrm{pH}_{\mathrm{H} 20}$ & & 5,4 & Fe ext. & $\left(\mathrm{mg} \mathrm{kg}^{-1}\right)$ & 8 \\
\hline Nec. Cal & $\left(\mathrm{t} \mathrm{CaCO}_{3} \mathrm{ha}^{-1}\right)$ & 2 & Mn ext. & $\left(\mathrm{mg} \mathrm{kg}^{-1}\right)$ & $<2,5$ \\
\hline $\mathrm{N}$ total & $\left(\mathrm{g} \mathrm{kg}^{-1}\right)$ & 0,25 & Zn ext. & $\left(\mathrm{mg} \mathrm{kg}^{-1}\right)$ & $<0,10$ \\
\hline $\mathrm{CE}$ & $\left(\mathrm{mS} \mathrm{cm} \mathrm{cm}^{-1}\right)$ & 0,01 & $\mathrm{Cu}$ ext. & $\left(\mathrm{mg} \mathrm{kg}^{-1}\right)$ & 2,7 \\
\hline MO & $\left(\mathrm{g} \mathrm{kg}^{-1}\right)$ & 3,5 & B ext. & $\left(\mathrm{mg} \mathrm{kg}^{-1}\right)$ & $<0,20$ \\
\hline P ext. & $\left(\mathrm{mg} \mathrm{kg}^{-1}\right)$ & $<23$ & Cd "total" & $\left(\mathrm{mg} \mathrm{kg}^{-1}\right)$ & $<0,05$ \\
\hline K ext. & $\left(\mathrm{mg} \mathrm{kg}^{-1}\right)$ & $<24$ & $\mathrm{Cu}$ "total" & $\left(\mathrm{mg} \mathrm{kg}^{-1}\right)$ & 14,6 \\
\hline Mg ext & $\left(\mathrm{mg} \mathrm{kg}^{-1}\right)$ & 17 & Ní "total" & $\left(\mathrm{mg} \mathrm{kg}^{-1}\right)$ & 1,8 \\
\hline Bases de troca & & & $\mathrm{Pb}$ "total" & $\left(\mathrm{mg} \mathrm{kg}^{-1}\right)$ & 2,8 \\
\hline $\mathrm{Ca}$ & $\left(\mathrm{cmol}^{(+)} \mathrm{kg}^{-1}\right)$ & 0,41 & Zn "total" & $\left(\mathrm{mg} \mathrm{kg}^{-1}\right)$ & 3,6 \\
\hline $\mathrm{Mg}$ & $\left(\mathrm{cmol}(+) \mathrm{kg}^{-1}\right)$ & 0,14 & $\mathrm{Hg}$ "total" & $\left(\mathrm{mg} \mathrm{kg}^{-1}\right)$ & 0,006 \\
\hline K & $\left(\mathrm{cmol}(+) \mathrm{kg}^{-1}\right)$ & 0,03 & Cr "total" & $\left(\mathrm{mg} \mathrm{kg}^{-1}\right)$ & 1,9 \\
\hline $\mathrm{Na}$ & $\left(\mathrm{cmol}(+) \mathrm{kg}^{-1}\right)$ & 0,01 & $\mathrm{MV}_{\mathrm{ap}}$ & $\left(\mathrm{g} \mathrm{cm}^{-3}\right)$ & 1,65 \\
\hline
\end{tabular}

Nec. Cal - necessidade de cal ; CE - condutividade elétrica ; MO - matéria orgânica; ext. - extraível; SBT - soma das bases de troca ; CTC - capacidade de troca catiónica ; GSB - grau de saturação de bases; MVap - massa volúmica aparente 
$(0-20 \mathrm{~cm})$ de um Arenossolo dístrico - ARdy, segundo a classificação de IUSS Working Group WRB (2015), da região de Alpiarça $\left(39^{\circ} 13^{\prime} 00^{\prime \prime} \mathrm{N}, 08^{\circ} 33^{\prime} 20^{\prime \prime} \mathrm{W}\right)$, cujas principais características físico-químicas se apresentam no Quadro 1.

Trata-se de um solo arenoso, muito pobre em matéria orgânica e em nutrientes, ácido, pouco saturado e de muito baixa capacidade de troca catiónica sendo, por isso, um tipo de solo adequado à realização de ensaios em vaso, em que se pretende obter resultados da aplicação de fertilizantes evidentes e num curto período de tempo.

Os teores de metais pesados determinados neste solo são inferiores aos limites máximos estabelecidos no DL n.․ 276/2009, de 2 de outubro, que estabelece as normas de utilização de lamas de depuração em solos agrícolas (MAOTDR, 2009) permitindo, assim, a incorporação deste tipo de produtos.

A lama aplicada era proveniente da ETAR de Beirolas, que é caracterizada por tratar efluentes urbanos e industriais. O Quadro 2 apresenta a composição da lama da ETAR utilizada, determinada no Laboratório Químico Agrícola Rebelo da Silva (LQARS), do INIAV.

No ensaio foi utilizado o milho (Zea mays L.), como planta teste, usando uma variedade de ciclo curto e de desenvolvimento rápido, tendo sido efetuado o corte das plantas 45 dias após a sementeira. Após desbaste, mantiveram-se quatro plantas por vaso. Durante o ensaio, a terra dos vasos foi mantida a $70 \%$ da capacidade máxima de retenção de água.

De modo a evitar a ocorrência de outros fatores limitantes ao desenvolvimento das plantas, antes da sementeira foi efetuada uma fertilização mineral de base, igual em todos os vasos, veiculando os nutrientes $\mathrm{N}, \mathrm{K}, \mathrm{Mg}, \mathrm{S}, \mathrm{Fe}, \mathrm{Mn}, \mathrm{Zn}, \mathrm{Cu}$ e B. A aplicação de azoto, na forma de nitrato de amónio, foi fracionada em três aplicações, espaçadas de duas a três semanas entre elas.

No ensaio, delineado em blocos completos casualizados com três repetições, foram considerados 13 tratamentos experimentais (T1 a T13). Um tratamento testemunha, sem aplicação de fósforo, quer através da lama quer de fosfato de cálcio, quatro tratamentos com níveis crescentes de lama (T2 a T5 correspondendo, respetivamente, a valores equivalentes a 30, 60, 90 e $120 \mathrm{t} \mathrm{ha}^{-1}$ ), quatro tratamentos com níveis crescentes de fosfato de cálcio, com um grau de pureza pro analise (T6 a T9) e, por fim, quatro tratamentos compostos por níveis crescentes de lama e fosfato de cálcio (Quadro 3). $\mathrm{O}$ ensaio decorreu sob ambiente controlado no Horto Químico Agrícola Boaventura de Azevedo, utilizando vasos de polietileno branco preenchidos com $4,5 \mathrm{~kg}$ de terra.

Quadro 2 - Caracterização da lama desidratada, da ETAR de Beirolas, utilizada no ensaio

\begin{tabular}{crcccc}
\hline Parâmetros & \multicolumn{5}{c}{ Parâmetros } \\
\hline $\mathrm{MS}$ & $(\%)$ & 19 & $\mathrm{Ni}$ & $\left(\mathrm{mg} \mathrm{kg}^{-1}\right)$ & 43 \\
$\mathrm{pH} \mathrm{H}_{20}$ & & 12 & $\mathrm{Zn}$ & $\left(\mathrm{mg} \mathrm{kg}^{-1}\right)$ & 680 \\
$\mathrm{CE}$ & $\left(\mathrm{mS} \mathrm{cm}^{-1}\right)$ & 2,0 & $\mathrm{Cr}$ & $\left(\mathrm{mg} \mathrm{kg}^{-1}\right)$ & 18,5 \\
$\mathrm{MO}$ & $(\%)$ & 64,4 & $\mathrm{~Pb}$ & $\left(\mathrm{mg} \mathrm{kg}^{-1}\right)$ & 20,6 \\
$\mathrm{~N}$ total & $(\%)$ & 7,39 & $\mathrm{Cd}$ & $\left(\mathrm{mg} \mathrm{kg}^{-1}\right)$ & 2,8 \\
$\mathrm{P}$ & $\left(\mathrm{mg} \mathrm{kg}^{-1}\right)$ & 15590 & $\mathrm{Cu}$ & $\left(\mathrm{mg} \mathrm{kg}^{-1}\right)$ & 157 \\
$\mathrm{~K}$ & $\left(\mathrm{mg} \mathrm{kg}^{-1}\right)$ & 1909 & $\mathrm{Hg}$ & & 1 \\
$\mathrm{Ca}$ & $\left(\mathrm{mg} \mathrm{kg}^{-1}\right)$ & 87336 & $\mathrm{C} / \mathrm{N}$ & & 4,8 \\
$\mathrm{Mg}$ & $\left(\mathrm{mg} \mathrm{kg}^{-1}\right)$ & 3558 & E. coli & (células/g de MF) & $<1000$ \\
$\mathrm{Na}$ & $\left(\mathrm{mg} \mathrm{kg}^{-1}\right)$ & 1200 & Salmonella spp. & (células/g de MF) & ausente \\
\hline
\end{tabular}

Valores referidos à matéria seca (MS), com exceção do pH, CE e E. coli e Salmonella. 
Quadro 3 - Quantidade de fósforo (P) veículado pela lama (L) e/ou pelo fosfato de cálcio (F) adicionada ao solo em cada tratamento experimental

\begin{tabular}{ccccc}
\hline $\begin{array}{c}\text { Tratamentos } \\
\text { experi- } \\
\text { mentais }\end{array}$ & $\begin{array}{c}\text { Nível } \\
\text { de P }\end{array}$ & $\begin{array}{c}\text { P veiculado } \\
\text { pela lama } \\
\text { (g/vaso) }\end{array}$ & $\begin{array}{c}\text { P veiculado } \\
\text { pelo fosfato } \\
\text { de cálcio } \\
\text { (g/vaso) }\end{array}$ & $\begin{array}{c}\text { P total } \\
\text { (g /vaso) }\end{array}$ \\
\hline T1 & 0 & 0 & 0 & 0 \\
T2 & 1 L & 0,121 & 0 & 0,121 \\
T3 & 2 L & 0,245 & 0 & 0,245 \\
T4 & 3 L & 0,364 & 0 & 0,364 \\
T5 & 4 L & 0,485 & 0 & 0,485 \\
T6 & 1 F & 0 & 0,121 & 0,121 \\
T7 & 2 F & 0 & 0,245 & 0,245 \\
T8 & 3 F & 0 & 0,364 & 0,364 \\
T9 & 4 F & 0 & 0,485 & 0,485 \\
T10 & $1 / 2$ L+ 1/2F & 0,061 & 0,061 & 0,122 \\
T11 & 1L + 1 F & 0,121 & 0,121 & 0,242 \\
T12 & $3 / 2 \mathrm{~L}+3 / 2 F$ & 0,182 & 0,182 & 0,364 \\
T13 & 2L + 2F & 0,245 & 0,245 & 0,490 \\
\hline
\end{tabular}

\section{Métodos analíticos}

As análises efetuadas ao solo e ao material vegetal foram realizadas com base nos métodos laboratoriais utilizados no INIAV - Laboratório Químico Agrícola Rebelo da Silva (INIAV, I.P., 2016). As análises efetuadas à lama regeram-se pelos parâmetros e normas de referência estipulados no DL n.․․ 276/2009 (MAOTDR, 2009).

Para a análise estatística dos resultados experimentais, recorreu-se à folha de cálculo Excel e ao programa de análise estatística Statgraphics Plus (versão 5.1), tendo sido utilizados o método de análise de variância (ANOVA, Tipo II) para avaliação do efeito dos diferentes tratamentos experimentais sobre as diversas variáveis controladas, o teste múltiplo de comparação de médias, método de Duncan $(\mathrm{p}=0,05)$, para comparação a posteriori das médias correspondentes às modalidades experimentais e análise de regressão, para avaliar a resposta da produção de biomassa à aplicação de quantidades crescentes de lama e a resposta do teor de $\mathrm{P}$ nos tecidos vegetais à aplicação de fósforo veiculado pela lama e/ou pelo fosfato de cálcio.

\section{RESULTADOS E DISCUSSÃO}

\section{Produção de biomassa}

Foi notória a resposta da produção de biomassa da parte aérea aos diferentes tratamentos experimentais, efeito altamente significativo $(p \leq 0,001)$, verificando-se distintas respostas de produção resultantes da aplicação de quantidades crescentes de fósforo veiculado pelas diferentes formas (Figura 1).

A produção mais baixa foi obtida no tratamento testemunha em que não foi aplicado fósforo, quer na forma mineral quer na forma de lama (T1).

Nos tratamentos experimentais em que apenas foi aplicada lama (T2 a T5) ocorreu um aumento significativo $(p=0,05)$ na produção até à aplicação de quantidade equivalente a $90 \mathrm{t} \mathrm{ha}^{-1}$ (T4), verificando-se um decréscimo significativo quando se aplicou a dose mais elevada, que corresponde a 120 t ha-1 $^{-1}$ (T5). Esta resposta demonstra a necessidade de estabelecer limites para as quantidades de lama a aplicar ao solo agrícola que deverão ser confirmadas através da realização de ensaios de fertilização com lamas nas diversas culturas em que haja interesse na sua utilização.

Outros autores, nomeadamente Domingues et al. (2003), observaram também que doses excessivas de lama produzem efeitos de quebra de produção das culturas, o que provavelmente estará associado à presença de agentes de fitotoxicidade que podem ser de diferente natureza. No caso em estudo, a lama utilizada possui baixos teores de metais pesados. Possivelmente terá ocorrido no solo um excesso de azoto amoniacal, veiculado pela lama, associado a um aumento da condutividade elétrica que, na dose mais elevada de lama, poderão ter afetado o desenvolvimento das plantas.

Para os restantes tratamentos experimentais, nos quais foi aplicado fosfato de cálcio (T6 a T9) e a mistura de lama com fosfato de cálcio (T10 a T13), o aumento da produção de biomassa acompanhou o aumento das quantidades de fósforo veiculadas pela forma mineral e/ou orgânica.

Pode-se observar, pelo Quadro 3 e pela Figura 1, que os tratamentos que receberam as quantidades mais 


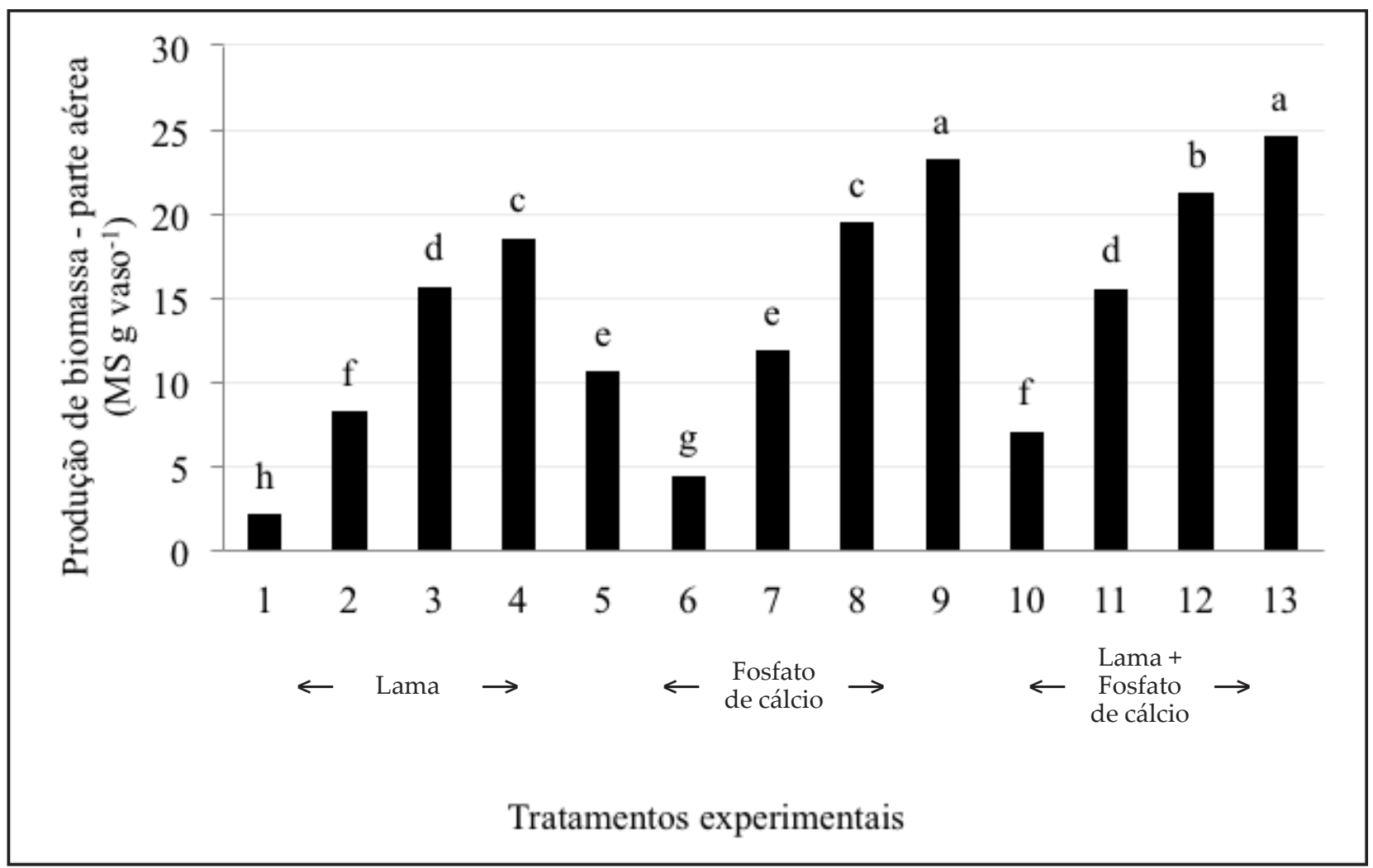

Figura 1 - Resposta da produção média de biomassa, da parte aérea, aos tratamentos experimentais.

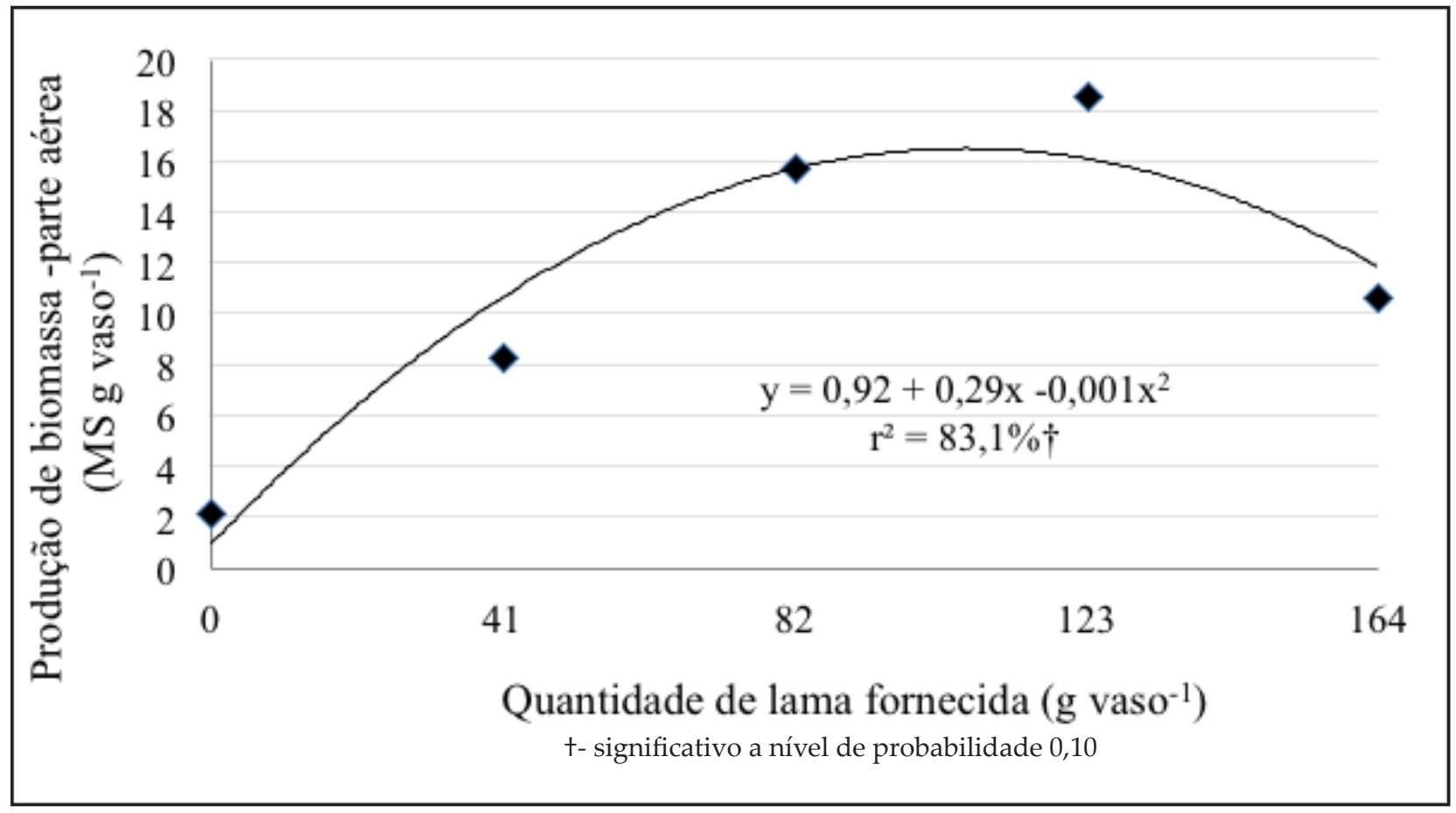

Figura 2 - Resposta da produção média de biomassa, parte aérea, à aplicação de quantidades crescentes de lama. 
elevadas de fósforo (equivalente a $355 \mathrm{~kg} \mathrm{P} \mathrm{ha}^{-1}$ ) veiculado pelo fosfato de cálcio (T9) e pela mistura deste com lama (T13) não diferem significativamente entre si, apresentando os resultados mais elevados de produção de biomassa da parte aérea.

A análise comparativa dos resultados obtidos nos tratamentos experimentais com fósforo, veiculado apenas na forma mineral, com os obtidos nos tratamentos em que este elemento foi veiculado em partes iguais na forma inorgânica e orgânica (lama), permitem concluir que se poderá substituir parte da adubação mineral fosfatada por lama, sem prejudicar a produção de biomassa.

Assim, para os valores médios de produção de biomassa da parte aérea ajustou-se uma equação de regressão em função da quantidade de lama aplicada aos vasos (Figura 2).

O modelo que melhor se ajusta aos resultados obtidos é uma função polinomial de 2.․o grau $(p<0,10)$, com um coeficiente de determinação de $83,1 \%$. De acordo com o modelo de regressão estimado, a aplicação de $105 \mathrm{~g}$ de lamas por vaso, o que corresponde aproximadamente a $77 \mathrm{t} \mathrm{ha}^{-1}$, conduziria à obtenção de uma produção máxima de biomassa, nas condições do ensaio.

Na Figura 3 é possível observar os resultados obtidos relativamente à produção de biomassa (parte aérea).

\section{Teores de fósforo nos tecidos vegetais}

O fósforo é um elemento essencial sendo o que, a seguir ao azoto, mais frequentemente limita a produção vegetal (Calouro, 2005). Este nutriente faz parte da estrutura de enzimas, proteínas, ácidos nucleicos e fosfolípidos, tendo um papel determinante na acumulação e transferência de energia que ocorrem em diversos fenómenos vitais para a planta (Malavolta, 1974; Jones et al., 1991; Varennes, 2003; Santos, 2015).

Apesar de os teores nas plantas terem sido de modo geral baixos, foi possível detetar um acréscimo significativo $(p=0,05)$ nos teores de fósforo nos tecidos vegetais com a aplicação de doses crescentes deste nutriente na forma de lama e/ou de fosfato de cálcio, apresentando valores significativamente $(p=0,05)$ mais elevados nos tratamentos experimentais que receberam a máxima quantidade de lama (T5) ou de fosfato de cálcio (T9), não havendo diferenças significativas $(p=0,05)$ entre eles.

Na Figura 4 representa-se a resposta do teor de fósforo nos tecidos vegetais à aplicação de quantidades crescentes do nutriente aplicado ao solo sob a forma de lama, fosfato de cálcio e da mistura de ambos. Verifica-se que as respostas foram lineares no caso da lama e da lama mais fosfato de cálcio, com coeficientes de determinação de $67,3 \%$ e $89,5 \%$, respetivamente.

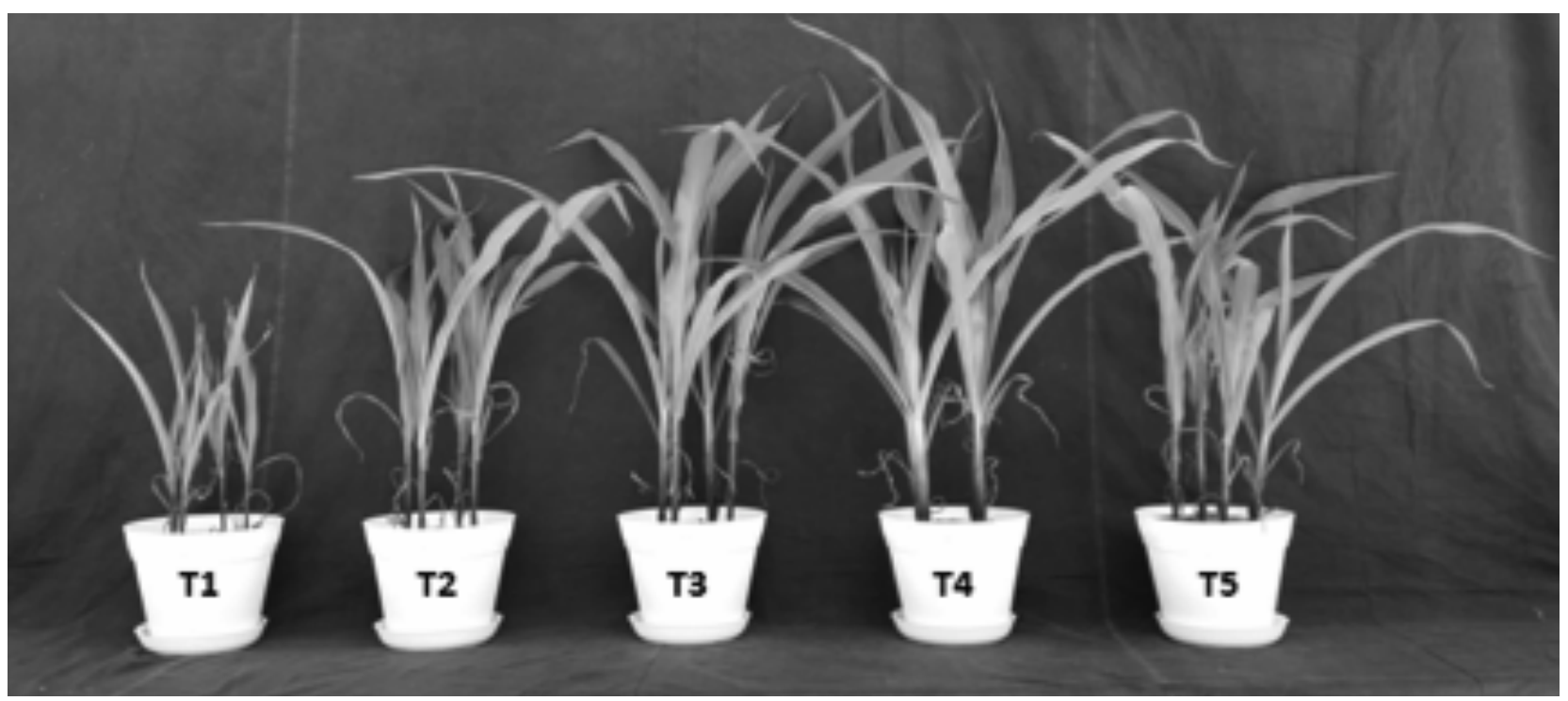

Figura 3 - Produção de biomassa, no final do ensaio, em função da aplicação de quantidades crescentes de lama. 


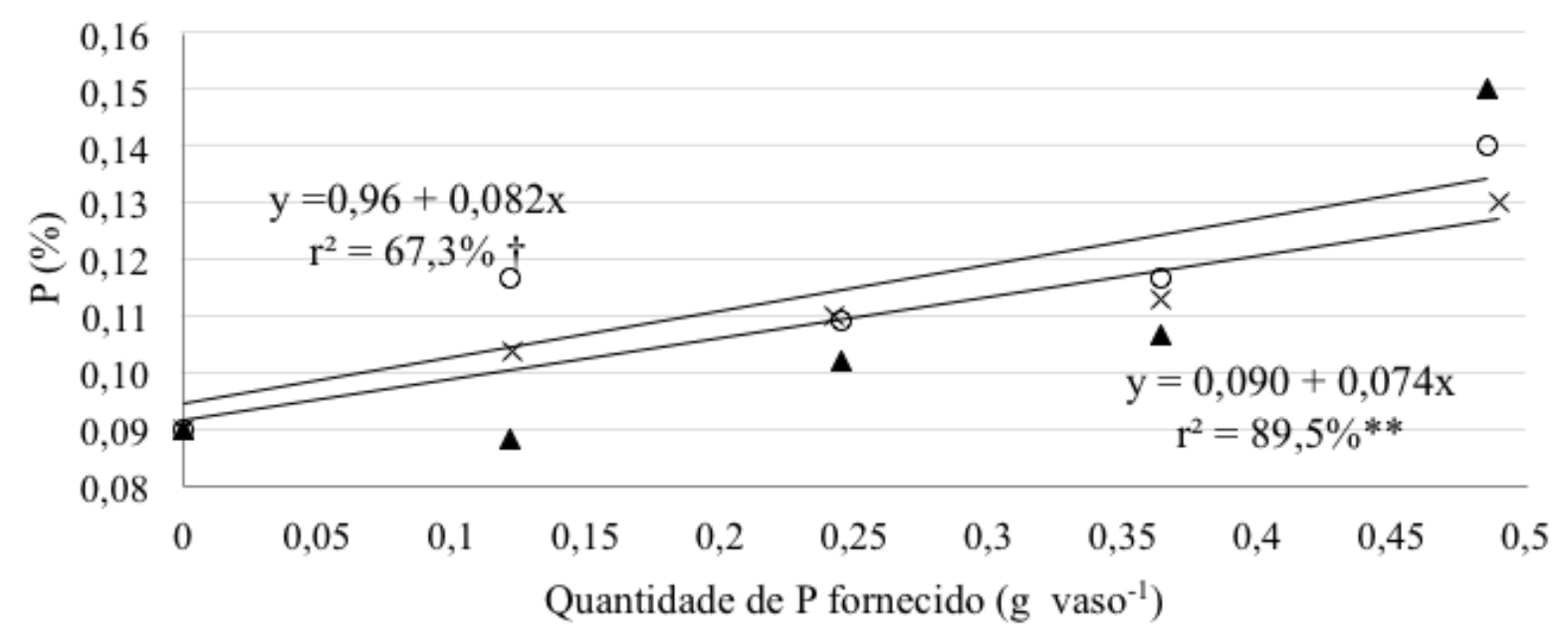

OLama $\quad \times_{\text {Lama }}+$ Fosfato de cálcio $\quad \boldsymbol{\Delta}_{\text {Fosfato de cálcio }}$

Figura 4 - Relação entre o teor de fósforo nos tecidos vegetais e a aplicação de quantidades crescentes de fósforo.

\section{Remoção e taxa de recuperação de fósforo}

Para todos os tratamentos experimentais, avaliou-se a remoção de fósforo total ( $\mathrm{P}$ rem) pelas plantas e a recuperação aparente de fósforo (RAP), através das seguintes fórmulas de cálculo propostas por Mengel e Kirkby (2001) (valores referidos à matéria seca):

$\mathrm{P}$ rem $=$ Produção de biomassa da parte aérea $\times[\mathrm{P}]$ da biomassa aérea

$\mathrm{RAP}=\left(\left(\mathrm{P}_{\text {rem }_{\mathrm{Tx}}}-\mathrm{P}\right.\right.$ rem $\left._{\mathrm{Tt}}\right) / \mathrm{P}$ aplicado $\left._{\mathrm{Tx}}\right) \times 100 \%$

Onde:

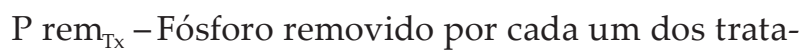
mentos experimentais;

$\mathrm{P}$ rem $_{\mathrm{Tt}}-$ Fósforo removido pelo tratamento testemunha.

Foram, assim, obtidos os valores que figuram no Quadro 4.
Quadro 4 - Fósforo removido (P rem) e recuperação aparente de fósforo (RAP) pela planta teste em cada um dos tratamentos experimentais

\begin{tabular}{|c|c|c|c|c|}
\hline \multicolumn{2}{|c|}{$\begin{array}{c}\text { Tratamentos } \\
\text { experimentais }\end{array}$} & \multirow{2}{*}{$\begin{array}{c}\begin{array}{c}\mathbf{P} \\
\text { adicionado } \\
\left.\text { (mg vaso- }^{-1}\right)\end{array} \\
0,000\end{array}$} & \multirow{2}{*}{$\begin{array}{c}\begin{array}{c}\text { P rem } \\
\left(\mathbf{m g ~ v a s o}^{-1}\right)\end{array} \\
1,9 \mathrm{~h}\end{array}$} & \multirow[t]{2}{*}{$\begin{array}{r}\text { RAP } \\
(\%)\end{array}$} \\
\hline$Q$ & $\mathrm{~T} 1$ & & & \\
\hline \multirow{4}{*}{$\begin{array}{l}\widetilde{\Xi} \\
\text { జ్త }\end{array}$} & $\mathrm{T} 2$ & 0,121 & $9,7 \mathrm{fg}$ & $6,4 \mathrm{ab}$ \\
\hline & T3 & 0,245 & $17,2 \mathrm{~d}$ & $6,2 \mathrm{ab}$ \\
\hline & $\mathrm{T} 4$ & 0,364 & $21,9 \mathrm{c}$ & $5,4 \mathrm{bc}$ \\
\hline & T5 & 0,485 & $14,7 \mathrm{de}$ & $2,7 \mathrm{e}$ \\
\hline \multirow{4}{*}{ 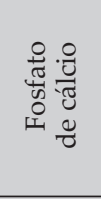 } & T6 & 0,121 & $4,0 \mathrm{~h}$ & $1,7 \mathrm{e}$ \\
\hline & $\mathrm{T} 7$ & 0,245 & 12,2 ef & $4,2 \mathrm{~d}$ \\
\hline & $\mathrm{T} 8$ & 0,364 & $21,2 \mathrm{c}$ & $5,3 \mathrm{bc}$ \\
\hline & T9 & 0,485 & $35,5 \mathrm{a}$ & $6,9 a$ \\
\hline \multirow{6}{*}{ 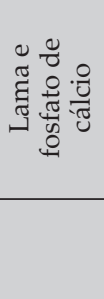 } & T10 & 0,121 & $7,2 \mathrm{~g}$ & $4,4 \mathrm{~cd}$ \\
\hline & $\mathrm{T} 11$ & 0,245 & $16,6 \mathrm{~d}$ & $6,0 \mathrm{ab}$ \\
\hline & $\mathrm{T} 12$ & 0,364 & $23,5 \mathrm{c}$ & $5,9 \mathrm{ab}$ \\
\hline & T13 & 0,485 & $32,4 \mathrm{~b}$ & $6,3 \mathrm{ab}$ \\
\hline & $\operatorname{sm}( \pm)$ & & 1,044 & 0,365 \\
\hline & c.v. $(\%)$ & & 10,8 & 12,3 \\
\hline
\end{tabular}

Resultados expressos na matéria seca a 100-105ㄷ; sm - desvio padrão da média; c.v. - coeficiente de variação; Resultados experimentais, na coluna, seguidos de letras iguais não diferem significativamente entre si $(p=0,05)$ 
A partir do Quadro 4 pode observar-se que foi nos tratamentos experimentais correspondentes às doses mais elevadas de fósforo veiculadas pelo fosfato de cálcio (T9) e pela conjugação desta forma mineral com a lama (T13) que foram removidas do solo pela planta teste as quantidades mais elevadas do nutriente (35,5 e 32,4 mg por vaso, respetivamente), apresentando valores significativamente superiores $(\mathrm{p}=0,05)$ aos dos outros tratamentos experimentais.

Atendendo aos resultados da recuperação aparente de $\mathrm{P}$ apurados, observou-se que quando se utilizaram doses crescentes de lama (T2 a T5), ocorreu um menor aproveitamento de fósforo pelas plantas, principalmente quando se aplicou a dose mais elevada (equivalente a $120 \mathrm{t} \mathrm{ha}^{-1}$ ). Já com a aplicação de fósforo unicamente na forma mineral (T6 a T9), houve um aproveitamento do nutriente crescente e significativo $(\mathrm{p}=0,05)$ com o aumento da quantidade aplicada.

Quando se utilizou simultaneamente fósforo na forma mineral e de lama, a recuperação deste elemento pela planta teste variou muito pouco com as doses aplicadas, apresentando valores estatisticamente iguais e elevados nos tratamentos T11, T12 e T13. Esta mistura promoveu, muito possivelmente, a mineralização do fósforo orgânico veiculado pela lama, ficando este, assim, mais disponível para a planta.

Os resultados obtidos estão de acordo com o previsto, uma vez que nos fertilizantes minerais os elementos, de forma geral, estão mais disponíveis para absorção pelas plantas do que nos fertilizantes orgânicos necessitando, neste caso, de ser previamente mineralizados para poderem ser absorvidos. Por outro lado, os resultados obtidos sugerem a existência de um efeito benéfico da presença de matéria orgânica sobre o aumento da disponibilidade deste nutriente, tal como é referido por vários autores (Brady e Weil, 2002; Santos, 2015), levando a concluir que, quando a lama é misturada com formas inorgânicas de fósforo (fosfato de cálcio, neste caso) é possível obter resultados equivalentes aos obtidos com a utilização de fertilizantes inorgânicos.

\section{CONCLUSÕES}

O trabalho desenvolvido permitiu estabelecer algumas conclusões, necessariamente indissociáveis das condições que as fundamentaram e, como tal, de generalização limitada. Os resultados obtidos revelaram a necessidade de se efetuarem mais estudos, aprofundando o tema abordado, mas permitiram retirar as seguintes conclusões:

A produção de biomassa da planta teste revelou elevada dependência da fertilização fosfatada, registando valores significativamente superiores nos tratamentos experimentais que receberam maiores quantidades de fósforo.

Demonstrou-se que o fósforo é, de modo geral, um elemento que apresenta valores de recuperação aparente muito baixos.

Confirmou-se a necessidade de estabelecer limites nas quantidades de lamas a fornecer ao solo agrícola, que podem não depender apenas do seu teor em metais pesados.

Os resultados da aplicação conjunta de lama com o fosfato de cálcio, observados quer sobre a produção da planta teste quer sobre a recuperação aparente de fósforo, permitem concluir que a lama poderá substituir em parte os fertilizantes minerais fosfatados já que a sua mistura permite obter resultados equivalentes aos obtidos apenas usando os tradicionais adubos inorgânicos. 


\section{REFERÊNCIAS BIBLIOGRÁFICAS}

Brady, N.C. \& Weil, R.R. (2002) - The Nature and Properties of Soil. Pearson Education, New Jersey. 960 p.

Calouro, F. (2005) - Atividades Agrícolas e Ambiente. Porto, 1.ㄹ ed. Sociedade Portuguesa de Inovação, 94 p. www.spi.pt/documents/books/agricultura ambiente/docs/Manualamb I.pdf

Cordell, D. \& White, S. (2014) - Life's Bottleneck: Sustaining the World's Phosphorus for a Food Secure Future. Annual Review of Environment and Resources, vol. 39, p. 161-188. http://dx.doi.org/10.1146/ annurev-environ-010213-113300

Domingues, H.; Monteiro, O.R.; Pedra, F.; Amaro, J.T. \& Gusmão, M.R. (2003) - Aplicação de lamas residuais urbanas em solos agrícolas. Síntese dos estudos desenvolvidos no Departamento de Ciências do Solo, da Estação agronómica Nacional - INIA. Revista de Ciências Agrárias, vol. 25, n. 3-4, p. 341-352.

Fytili, D. \& Zabaniotou, A. (2008) - Utilization of sewage sludge in EU applications and new methods - A review. Renewable and Sustainable Energy Reviews, vol. 12, n. 1, p. 116-140. https://doi.org/10.1016/j. rser.2006.05.014

Gonçalves, M.S. (2005) - Gestão de resíduos orgânicos. Porto, 1. ed. Sociedade Portuguesa de Inovação, 104 p. INIAV (2016) - Lista de determinações e métodos analíticos na Área de Ambiente e Recursos Naturais. Agosto 2016, 9 p. [cit. 2017-03-23]. http://www.iniav.pt/fotos/editor2/mod lqars 82 lista analises efetuadas arn 08 2016.pdf

IUSS Working Group WRB (2015) - World Reference Base for Soil Resources 2014. Update 2015. International soil classification system for naming soils and creating legends for soil maps. Roma, World Soil Resources Report 106, 203 p. [cit. 2017-03-22]. http://www.fao.org/3/a-i3794e.pdf

Jones, J.B.; Wolf, J.B. \& Mills, H.A. (1991) - Plant analysis handbook. Micro-Macro Publishing, Estados Unidos da América. 213 p.

Malavolta, E.; Haag, H.P.; Mello, F.A.F. \& Brasil Sobro , M.O.C. (1974) - Nutrição Mineral e Adubação de Plantas Cultivadas. Livraria Pioneira Editora, São Paulo. 727 p.

MAOTDR (2009) - DL n. 276/2009, de 02 de outubro. Diário da República n. 192 - I Série. Ministério do Ambiente, do Ordenamento do Território e do Desenvolvimento Regional. Lisboa, p. 7154-7165. www. drapc.min-agricultura.pt/base/legislacao/files/decreto lei 276 2009.pdf

Mengel, K. \& Kirkby, E.A. (2001) - Principles of plant nutrition. 5th ed., Kluwer academic Publishers, Dordrecht, Netherlands. 849 p.

Santos, J.Q. (2015) - Fertilização. Fundamentos agroambientais da utilização dos adubos e corretivos. Publindustria, Ed. Técnicas, Lisboa. 537 p.

Soveral-Dias, J.C. (2004) - Guia de Boas Práticas - Aplicação de Lamas na Agricultura. Lisboa, Reciclamas Multigestão Ambiental, S.A., 159 p.

Varennes, A. (2003) - Produtividade dos solos e Ambiente. Escolar Editora, Lisboa. 490 p. 